\title{
TrkB Signaling in Dorsal Raphe Nucleus is Essential for Antidepressant Efficacy and Normal Aggression Behavior
}

\author{
Megumi Adachi',2, Anita E Autry',2, Melissa Mahgoub', Kanzo Suzuki' and Lisa M Monteggia*,I \\ 'Department of Neuroscience, The University of Texas Southwestern Medical Center, Dallas, TX, USA
}

Brain-derived neurotrophic factor (BDNF) and its high affinity receptor, tropomyosin receptor kinase B (TrkB), have important roles in neural plasticity and are required for antidepressant efficacy. Studies examining the role of BDNF-TrkB signaling in depression and antidepressant efficacy have largely focused on the limbic system, leaving it unclear whether this signaling is important in other brain regions. BDNF and TrkB are both highly expressed in the dorsal raphe nucleus (DRN), a brain region that has been suggested to have a role in depression and antidepressant action, although it is unknown whether BDNF and TrkB in the dorsal raphe nucleus are involved in these processes. We combined the adeno-associated virus (AAV) with the Cre-loxP site-specific recombination system to selectively knock down either Bdnf or TrkB in the DRN. These mice were then characterized in several behavioral paradigms including measures of depression-related behavior and antidepressant efficacy. We show that knockdown of TrkB, but not Bdnf, in the DRN results in loss of antidepressant efficacy and increased aggression-related behavior. We also show that knockdown of TrkB or Bdnf in this brain region does not have an impact on weight, activity levels, anxiety, or depression-related behaviors. These data reveal a critical role for TrkB signaling in the DRN in mediating antidepressant responses and normal aggression behavior. The results also suggest a non-cell autonomous role for $\mathrm{BDNF}$ in the DRN in mediating antidepressant efficacy.

Neuropsychopharmacology (2017) 42, 886-894; doi:I0.1038/npp.2016.20I; published online 19 October 2016

\section{INTRODUCTION}

Major depressive disorder (MDD) is a leading cause of disability worldwide and affects $\sim 7 \%$ of the US population annually (Kessler et al, 2003). Symptoms of MDD include anhedonia, persistent feelings of guilt or sadness, and alterations in appetite and activity levels. Concordance rates indicate $\mathrm{a} \sim 50 \%$ genetic contribution to MDD, although no specific genes have been identified as causative for the disorder (Lesch, 2004). Pharmacological treatments for MDD include selective serotonin reuptake inhibitors (SSRIs), norepinephrine reuptake inhibitors, tricyclic antidepressants, and monoamine oxidase inhibitors, all of which target the monoaminergic system. These antidepressants are thought to rapidly increase monoamine levels at the synapse; however, the delay in onset of efficacy suggests that downstream molecular alterations may be responsible for the antidepressant effects (Bjorkholm and Monteggia, 2015; Lindholm and Castren, 2014).

A potential downstream target of antidepressant treatment is brain-derived neurotrophic factor (BDNF). BDNF is the most prevalent neurotrophin in the central nervous system

\footnotetext{
* Correspondence: LM Monteggia, Department of Neuroscience, The University of Texas Southwestern Medical Center, 5323 Harry Hines Boulevard, NB4, Dallas, TX 75390-9III, USA. Tel: +2I4 648 5548, Fax: +2I4 648 4947, E-mail: lisa.monteggia@utsouthwestern.edu

${ }^{2}$ These authors contributed equally to this work.

Received I June 2016; revised 5 August 2016; accepted 2 September 2016; accepted article preview online 16 September 2016
}

(CNS) and binds to its high-affinity receptor, tropomyosin receptor kinase B (TrkB), to exert effects on neuronal development, survival, and learning and memory (Waterhouse and $\mathrm{Xu}, 2009$ ). In depressed patients, BDNF expression is decreased in the hippocampus, a brain region important for mood regulation (Castren and Rantamaki, 2010). Conversely, BDNF expression is increased in the hippocampus after chronic, but not acute, antidepressant treatment (Duman and Monteggia, 2006). Collectively, these studies have contributed to the hypothesis that a decrease in BDNF expression may mediate depression-related behavior and, conversely, an increase in BDNF expression in the hippocampus is necessary for antidepressants to mediate their therapeutic effect (Duman and Monteggia, 2006).

The direct link between the role of BDNF and depression has not been as straightforward as the data linking BDNF to antidepressant responses. Preclinical studies have shown infusion of BDNF into the midbrain (Hoshaw et al, 2005) or hippocampal regions (Shirayama et al, 2002) is sufficient to produce antidepressant-like effects in rodents. Consistent with these results, selective deletion of BDNF in forebrain regions attenuates the action of antidepressants, demonstrating that BDNF is necessary for antidepressant efficacy (Monteggia et al, 2004). In previous work, we examined whether the hippocampus was an essential site for BDNF action in antidepressant responses using a viral-mediated approach to selectively delete $B d n f$ in sub-regions of the hippocampus (Adachi et al, 2008). We found that selective deletion of Bdnf in the dentate gyrus, but not the CA1 
sub-region, results in attenuated antidepressant-like responses demonstrating a region-specific effect of BDNF in mediating antidepressant efficacy (Adachi et al, 2008).

The dentate gyrus receives strong serotonergic input from the dorsal raphe nucleus (DRN) (Haenisch et al, 2009; Tork, 1990). Serotonin neurons in the DRN are thought to be essential in mood behavior and antidepressant responses (McDevitt and Neumaier, 2011), and express BDNF and its high-affinity receptor TrkB (Lunden and Kirby, 2013; Madhav et al, 2001). BDNF can directly influence serotonergic neurons by increasing serotonin synthesis and activity of these neurons (Siuciak et al, 1996; Siuciak et al, 1998). Conversely, antidepressants that increase serotonin levels can increase BDNF expression in forebrain regions (Altar et al, 2003; Nibuya et al, 1995). Although these studies demonstrate interactions between BDNF and the serotonergic system, there can be regional specificity effects. Previous work has shown that loss of BDNF in forebrain regions attenuates serotonin-1A receptor function in hippocampus but not in dorsal or median raphe (Burke et al, 2013; Hensler et al, 2007). Building on our previous work demonstrating a requirement for $\mathrm{BDNF}$ in the dentate gyrus for antidepressant responses, in this study we examined whether BDNF-TrkB signaling in the DRN was also required for antidepressant efficacy. To address this question, we used a viral strategy to selectively express Cre recombinase in the DRN of adult floxed $B d n f$ (flBdnf) or floxed TrkB (flTrkB) mice and examined the impact on antidepressant responses.

\section{MATERIALS AND METHODS}

\section{Mice}

Mice were housed in a vivarium on a $12 \mathrm{~h}$ light/dark cycle with access to food and water ad libitum. The floxed $B d n f$ $(\mathrm{flBdnf})$ and floxed $\operatorname{TrkB}(\mathrm{flTrkB})$ mice were generated as previously described and maintained as homozygous crosses (Luikart et al, 2005; Rios et al, 2001). Experiments used 3- to 5-month-old male mice (age and weight-matched littermates) similar to those used in the previous study examining the contribution of BDNF in hippocampal subregions to antidepressant responses (Adachi et al, 2008). Animal procedures were approved by the Institutional Animal Care and Use Committee at UT Southwestern Medical Center in compliance with US Public Health Service guidelines.

\section{Adeno-Associated Virus}

Adeno-associated virus (AAV) expressing green fluorescent protein (GFP) tagged to Cre recombinase (AAV-GFP-Cre), or the control AAV-GFP, were obtained from the Penn Vector Core; AAV-GFP-Cre and AAV-GFP are AAV2/1. CMV.HI.GFP-Cre.SV40 and AAV2/1.CMV.PI.EGFP.WPRE. bGH, respectively. Previous work with the AAV-GFP-Cre has shown that GFP does not interfere with Cre recombinase activity (Adachi et al, 2008).

\section{Surgery}

Animals were anesthetized by intraperitoneal (i.p.) injection of $100 \mathrm{mg} / \mathrm{kg}$ ketamine and $10 \mathrm{mg} / \mathrm{kg}$ xylazine, and mounted in a stereotaxic apparatus. Holes were drilled above the injection sites on the exposed skull. Coordinates relative to lambda for the DRN were as follows: $\mathrm{AP}-0.4$; $\mathrm{ML} \pm 0.6$; DV -2.8 with needle at a $14^{\circ}$ angle from the AP axis. A Hamilton syringe with a 33-gauge needle delivered $1 \mu \mathrm{l}$ of either AAV-GFP or AAV-GFP-Cre virus over $4 \mathrm{~min}$ and left an additional $5 \mathrm{~min}$ for complete diffusion of virus.

\section{Quantitative Reverse-Transcription PCR}

On completion of the behavioral studies, mice were killed and the brains dissected, rapidly frozen on dry ice, and stored at $-80^{\circ} \mathrm{C}$. Brains were sectioned at $14 \mu \mathrm{m}$ on a cryostat and mounted on PEN membrane-coated slides, then briefly dehydrated in a series of ethanol solution $(70,90$, and $100 \%)$ for laser microdissection. DRN were identified by GFP epifluorescence and microdissected out using an AS LMD system (Leica, Bannockburn, IL). Sections (7-8/mouse; $\sim 110 \mu \mathrm{m}$ apart between sections) were pooled per brain, encompassing the majority of the AAV injection site. RNA from DRN was extracted using the PicoPure RNA isolation kit (Arcturus, Mountain View, CA), treated with DNaseI for $30 \mathrm{~min}$ at $37^{\circ} \mathrm{C}$, and reverse transcribed by using random hexamers and reverse transcriptase III (Invitrogen, Carlsbad, CA) to synthesize single-stranded $\mathrm{cDNA}$. Using $\mathrm{cDNA}$ as a template, transcripts for $B d n f$, TrkB, Cre recombinase, and Gapdh were amplified using Power SYBR Green PCR master mix (Applied Biosystems, Foster City, CA). The fold change in $C r e, T r k B$, and $B d n f$ expression relative to Gapdh was calculated as the mean \pm SEM.

\section{Behavioral Overview}

After surgery, mice recovered for 21 days, to ensure viral expression and recombination. Mice were monitored and weighed weekly to determine whether the gene deletion caused gross health problems. Mice were allowed to habituate to the behavior rooms for at least $1 \mathrm{~h}$ before testing. Behavioral testing was carried out one paradigm per day in order from least to most stressful paradigm (locomotor activity, open field, sucrose consumption, forced swim, and resident intruder tests). All experiments were performed by an individual blind to group/treatment assignment.

\section{Locomotor Activity}

A mouse was placed in a standard home cage and locomotor activity was recorded for $2 \mathrm{~h}$ under red light by photocell beams linked to computer acquisition software (San Diego Instruments, San Diego, CA).

\section{Open-Field Test}

Mice were placed in a $44 \mathrm{~cm}^{2}$ open field under dim lighting (40 lux) and their activity was monitored for $10 \mathrm{~min}$ with a video tracking system using Ethovision software. The time spent in the complete center and periphery $(5 \mathrm{~cm}$ around perimeters) of the open field were analyzed. 


\section{Sucrose Consumption Test}

The sucrose consumption test was modified from Gourley et al (2008) and carried out as described by Autry et al (2009). Before the test, mice were habituated to a $1 \%$ sucrose/ tap water solution for $48 \mathrm{~h}$. The mice were then deprived from water for 4,14 and $19 \mathrm{~h}$ on day 1,2 , and 3 , respectively. Immediately after the water deprivation, mice were temporarily housed in separate cages and allowed to access the sucrose solution for $1 \mathrm{~h}$. Sucrose intake was determined on day 3 by measuring the weight of the solution before and after the presentation of the sucrose solution. On day 4 , the mice were deprived from water for $19 \mathrm{~h}$, followed by $1 \mathrm{~h}$ presentation of water to assess water intake. Sucrose consumption is expressed as percentage sucrose intake (the amount of sucrose intake (from day 3)/total amounts of liquid intake (sucrose from day $3+$ water from day 4$)$ ).

\section{Forced Swim Test}

The forced swim test (FST) was performed as previously described (Porsolt et al, 1977). Briefly, mice were placed in a $4 \mathrm{~L}$ beaker containing $3 \mathrm{~L}$ of water at $22-24^{\circ} \mathrm{C}$ and behavior videotaped for $6 \mathrm{~min}$. The time spent immobile during the last $4 \mathrm{~min}$ of the test was scored by an observer blind to group assignments. Saline or desipramine $(15,15$, and $20 \mathrm{mg} / \mathrm{kg}$, respectively) was administered subchronically at 24,4 , and $1 \mathrm{~h}$ before the testing. For chronic fluoxetine treatment, a separate cohort of flTrkB animals underwent surgery to receive either AAV-GFP or AAV-GFP-Cre into the DRN. Twenty-one days after the surgery, the animals were subjected to the FST after subchronic treatments of fluoxetine $(10,10$, and $15 \mathrm{mg} / \mathrm{kg}$ at 24,4 , and $1 \mathrm{~h}$ before the testing, respectively). As this did not produce the antidepressant efficacy in the control animals (AAV-GFP), the animals were further subjected to a daily treatment of $10 \mathrm{mg} / \mathrm{kg}$ fluoxetine or saline delivered i.p. for 8 weeks, and the following day the animals were tested in the FST. The data were expressed as a ratio for each animal: (time spent immobile from FST after 8 weeks treatment)/(time spent immobile from FST after subchronic treatment).

\section{Resident Intruder Test}

The resident intruder test was performed as described by Monteggia et al (2004). One week after the FST, mice were singly housed in home cages for 10 days without bedding changed. The animals had not previously received any drug treatment. On day 11, an adult male mouse was introduced in the cage for $5 \mathrm{~min}$ and the encounter was videotaped. The videotapes were scored for aggressive behavior including latency to attack and number of bite attacks, wrestling, chasing, tail rattling, and submissive posturing of the intruder mouse.

\section{Statistical Analysis}

All data are presented as mean \pm SEM. Statistical significance was determined by a Student's $t$-test for locomotor activity, open field, sucrose consumption, and resident intruder tests, as well as QRT-PCR analysis of Bdnf, TrkB and Cre mRNA. The FST was analyzed by two-way ANOVA.
A $p$-value of a $p \leq 0.05$ was considered statistically significant in all experiments.

\section{RESULTS}

\section{Localized Deletion of $B d n f$ in the DRN}

To delete $B d n f$ in a region-specific manner, we bilaterally injected AAV-GFP-Cre into the DRN of flBdnf mice (Figure 1a). This approach induces excision of the gene sequence flanked by the loxP sites specifically in the region of the AAV injection. As a control, we bilaterally injected AAVGFP into the DRN of flBdnf mice. Mice recovered for 21 days after injections, a time frame we have previously shown to be sufficient for Cre-mediated recombination to occur in the brain (Adachi et al, 2009; Adachi et al, 2008). We verified that the placement of the virus injections were spatially restricted to the DRN by assessing GFP expression and then determined the knockdown of the gene using QRT-PCR analyses. To quantitatively assess the knockdown of $B d n f$ expression, we laser micro-dissected the DRN infected with the virus, which was identified by its GFP epifluorescence, and extracted RNA for QRT-PCR analyses. In flBdnf mice injected with AAV-GFP-Cre, we detected robust expression of Cre recombinase in the DRN, whereas AAV-GFP injected mice did not show Cre expression (Figure 1b). In agreement with the Cre expression data, the AAV-GFP-Cre-injected $\mathrm{flBdnf}$ mice had a $\sim 78 \%$ reduction in $B d n f$ expression in comparison with mice injected with AAV-GFP (Figure 1c). Animals with off-target injections were excluded from the analysis of the behavioral data.

\section{Loss of Bdnf in the DRN Does Not have an Impact on Antidepressant Responses}

Mice injected with either AAV-GFP or AAV-GFP-Cre were indistinguishable in overall appearance throughout the experiments. To assess whether selective deletion of $B d n f$ in the DRN had an impact on body weight, mice were weighed every week for 8 weeks following surgery. The weight of the AAV-GFP- and AAV-GFP-Cre-injected mice did not significantly differ, suggesting that loss of $B d n f$ in the DRN did not have an impact on body mass (data not shown). To assess the effect of BDNF signaling in the DRN on locomotor activity, mice were placed in a standard home cage with photocell beams linked to computer acquisition software for $120 \mathrm{~min}$. Total locomotor activity was unchanged between flBdnf mice injected with either AAV-GFP or AAV-GFP-Cre (Figure 2a, inset). However, there was an increase in the activity of AAV-Cre-injected flBdnf mice during the first $5 \mathrm{~min}$ of the task compared with AAV-GFP mice (Figure 2a).

The open-field test was used to examine the effect of localized deletion of Bdnf in the DRN on anxiety-like behavior. In this paradigm, increased time spent in the center of an open-field arena is thought to represent reduced anxiety, as this parameter is sensitive to benzodiazepine treatment (Griebel et al, 2000). We found no alteration in time spent in the center or periphery of the open-field arena in AAV-GFP- versus AAV-GFP-Cre-injected flBdnf mice, suggesting the localized deletion of $B d n f$ in the DRN did not have an impact on anxiety-like behavior (Figure 2b). In 
a

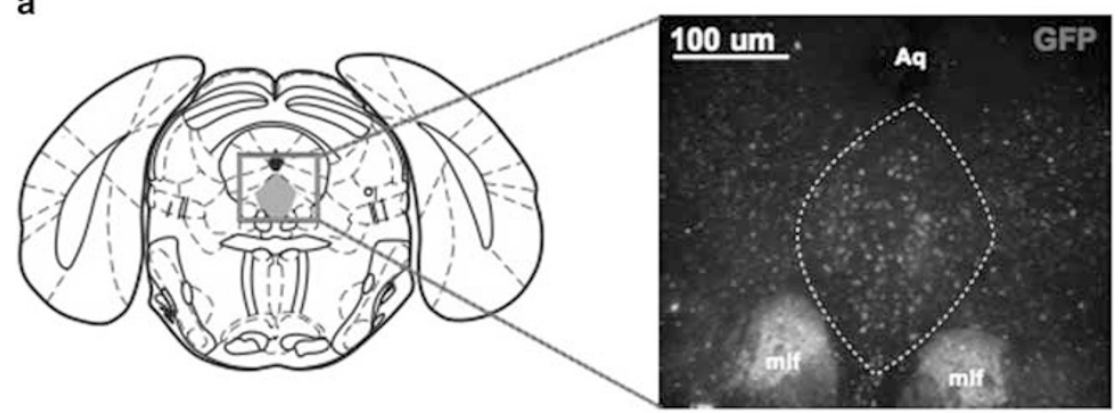

b

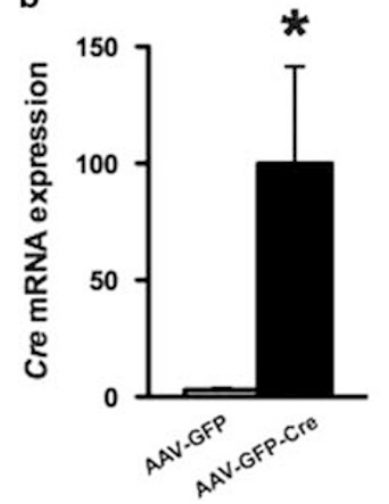

C

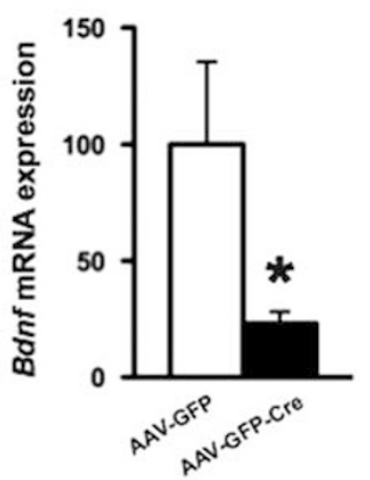

Figure I Localized knockdown of Bdnf in dorsal raphe nucleus (DRN). (a) Schematic of a coronal section containing DRN, highlighted in gray. (b, c) DRN containing green fluorescent protein (GFP) epifluorescence were laser micro-dissected and subjected to real-time PCR for quantification of Cre and Bdnf mRNA levels (adeno-associated virus expressing GFP (AAV-GFP), $N=12$; AAV-GFP tagged to Cre recombinase (AAV-GFP-Cre), $N=12$ ).

addition, there were no differences between distances traveled in the open field over the duration of the test (data not shown).

A previous study characterizing heterozygous $B d n f$ knockout mice suggested BDNF signaling in central serotonergic neurons may be linked to aggressive behavior (Lyons et al, 1999). Therefore, we investigated the impact of DRN-specific knockdown of Bdnf on aggressive behavior using the resident-intruder paradigm and quantified the latency to attack, as well as the number of attacks. We found that the loss of $B \mathrm{~d} n f$ in the DRN did not significantly alter latency to attack (Figure 2c) or number of attacks (data not shown) compared with flBdnf mice injected with AAV-GFP.

We assessed aspects of anhedonia using the sucrose consumption test. The amount of sucrose consumed was similar between flBdnf mice injected with either AAV-GFP or AAV-GFP-Cre, suggesting the localized deletion of BDNF in the DRN did not affect anhedonia-like behavior (Figure 2d). Although the loss of $B d n f$ in the DRN did not have an impact on the anhedonia aspect of depression, we examined whether it altered antidepressant efficacy using the FST. Antidepressant efficacy was assessed using the tricyclic antidepressant, desipramine, which increases serotonin and norepinephrine levels, and produces robust effects in the FST. There was no difference in immobility between vehicle-treated flBdnf mice injected with AAV-GFP or AAV-GFP-Cre virus (Figure 2e). However, after subchronic administration of desipramine, both the AAV-GFP- and AAV-GFP-Cre-injected flBdnf mice displayed significant decreases in immobility, suggestive of an antidepressant response as assessed by two-way ANOVA $\left(F_{1,30}=10.34\right.$, significant effect of drug with no genotype or interaction effect $\left.{ }^{*} ; p<0.05\right)$.

\section{Localized Deletion of TrkB in the DRN}

Our data, to this point, suggest that loss of BDNF in the DRN does not have an impact on several behavioral measures including antidepressant efficacy. As DRN neurons also express the high-affinity $\mathrm{BDNF}$ receptor, TrkB, we wondered whether TrkB signaling in the DRN is required to mediate antidepressant responses. Thus, we used the same experimental approach of injecting either AAV-GFP or AAV-GFPCre bilaterally in the DRN but now using adult flTrkB mice. Using QPCR, we found robust expression of Cre recombinase in mice injected with AAV-GFP-Cre, whereas Cre expression was absent in those injected with AAV-GFP (Figure 3a). In the same animals, TrkB mRNA was significantly reduced $\sim 30 \%$ within the DRN of flTrkB mice injected with AAV-GFP-Cre in comparison with those injected with AAV-GFP (Figure 3b).

\section{Selective Loss of TrkB and Antidepressant Responses}

The flTrkB mice injected with AAV-GFP were indistinguishable in appearance from those injected with AAV-GFP-Cre. To assess whether selective deletion of TrkB in the DRN had an impact on weight, mice were weighed weekly for 8 weeks 

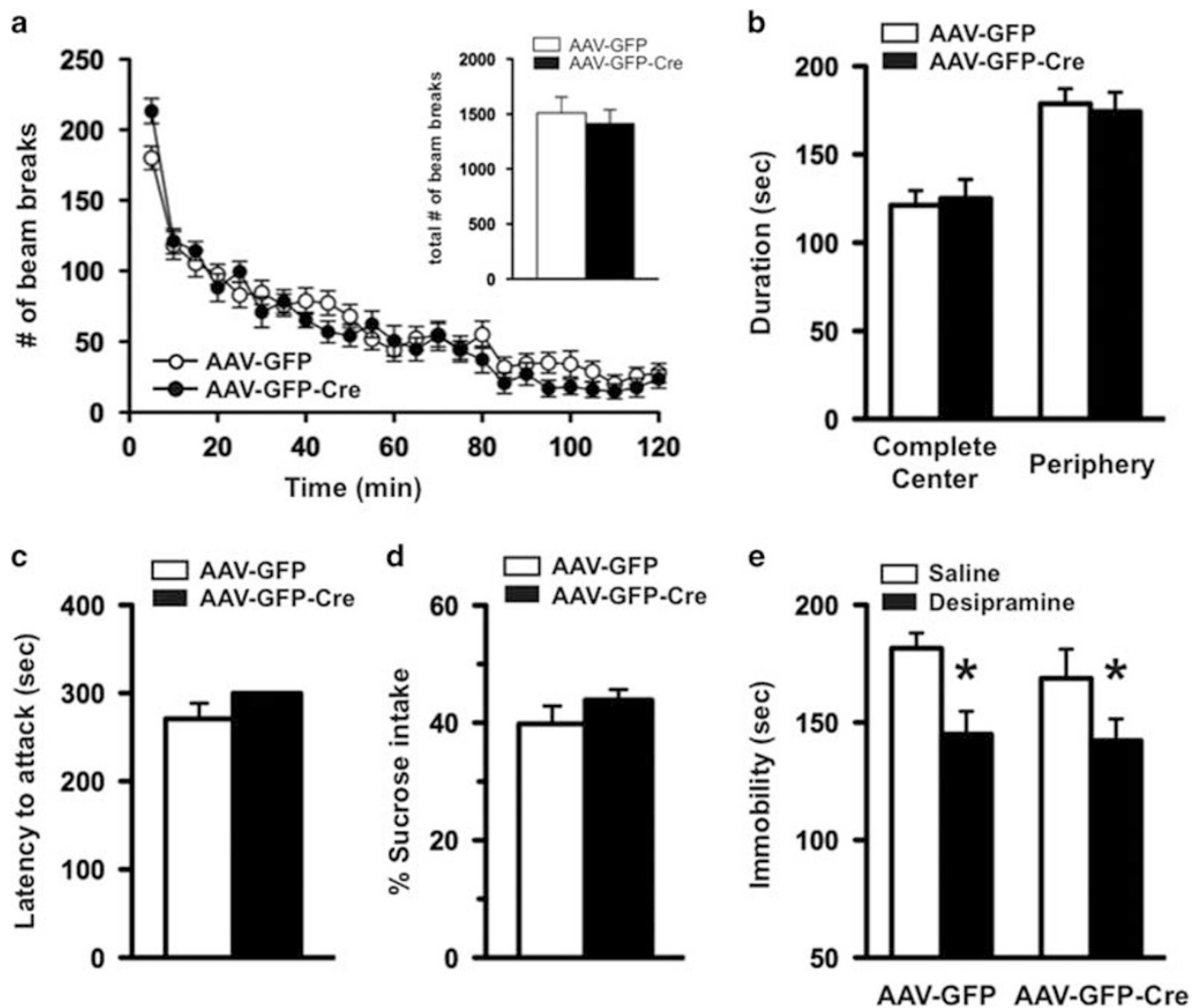

Figure 2 Mice with dorsal raphe nucleus (DRN)-specific Bdnf knockdown display overall normal behavioral phenotypes. (a) Mice injected with adenoassociated virus expressing GFP tagged to Cre recombinase (AAV-GFP-Cre) exhibited a significant increase in locomotor activity in the first 5 min, in comparison with those with AAV-GFP. However, the locomotor activity for the rest of $2 \mathrm{~h}$ testing period was similar between both groups of animals. The total number of beam breaks during the test period was indistinguishable between AAV-GFP and AAV-GFP-Cre mice (see the inset). (b) Anxiety-like behavioral phenotype was assessed by the open-field test. (c) In a resident-intruder paradigm, latency to attack a male intruder was measured in flBdnf mice injected with either AAV-GFP or AAV-GFP-Cre into DRN. (d) Depression-like behavior was assessed by the sucrose consumption test. (AAV-GFP, N = I2; AAV-GFP-Cre, $N=12$ for $a, b, c$, and d.) (e) Responses to an antidepressant, desipramine, were examined by the forced swim test (FST). In either AAV injection, sub-chronic treatment with desipramine resulted in significantly less immobile behavior in comparison with saline treatment, indicating a normal response to the antidepressant. (AAV-GFP/saline, N=9; AAV-GFP/desipramine, N=10; AAV-GFP-Cre/saline, N= 8; AAV-GFP-Cre/desipramine, N=7; twoway ANOVA $\left(F_{1}, 30\right)=10.34, p=0.0031$ for treatment followed by multiple comparison with $t$-test, $* p<0.05$.)
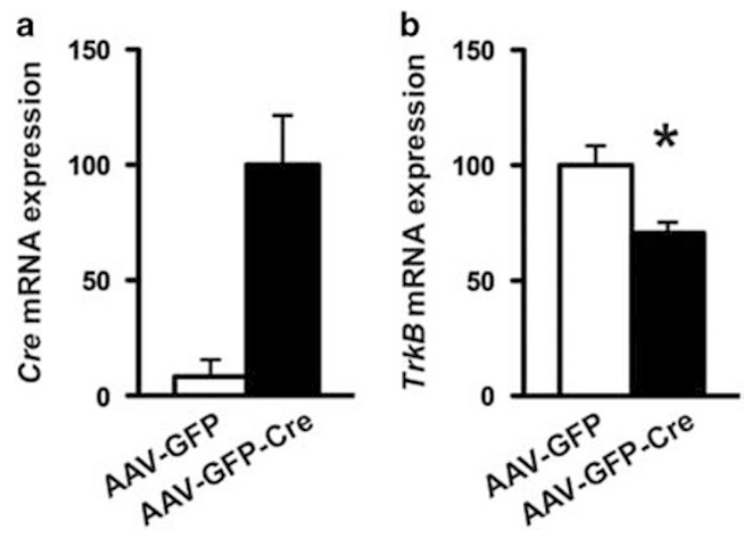

Figure 3 Localized knockdown of tropomyosin receptor kinase B (TrkB) in dorsal raphe nucleus (DRN). (a, b) DRN containing green fluorescent protein (GFP) epifluorescence were laser micro-dissected out and subjected to real-time PCR for quantification of (a) Cre and (b) TrkB mRNA levels. (Adeno-associated virus expressing GFP (AAV-GFP), $N=12 ;$ AAV-GFP tagged to Cre recombinase (AAV-GFP-Cre), $N=14$.) following surgery. The weight of the AAV-GFP- and AAVGFP-Cre-injected mice did not significantly differ following surgery, suggesting that loss of TrkB in the DRN did not have an impact on body mass (data not shown). Locomotor activity was assessed in the home-cage environment and comparable activity was found between mice injected with AAV-GFP or AAV-GFP-Cre during the $2 \mathrm{~h}$ test session, as well as when the data were analyzed in $5 \mathrm{~min}$ epochs (Figure 4a). In the open-field test, knockdown of TrkB in the DRN did not alter the time spent in the complete center or the periphery of the test area compared with the control mice, suggesting normal anxiety-like behavior (Figure $4 \mathrm{~b}$ ). Mice were also tested in the resident intruder paradigm and the loss of TrkB in the DRN resulted in a significantly shorter latency to attack compared to mice injected with AAV-GFP, suggesting an increase in aggressive behavior (Figure 4c). Mice were also examined in the sucrose consumption test. There was no significant difference in the amount of sucrose solution consumed between flTrkB mice injected with either AAV-GFP or AAV-GFP-Cre (Figure 4d). 

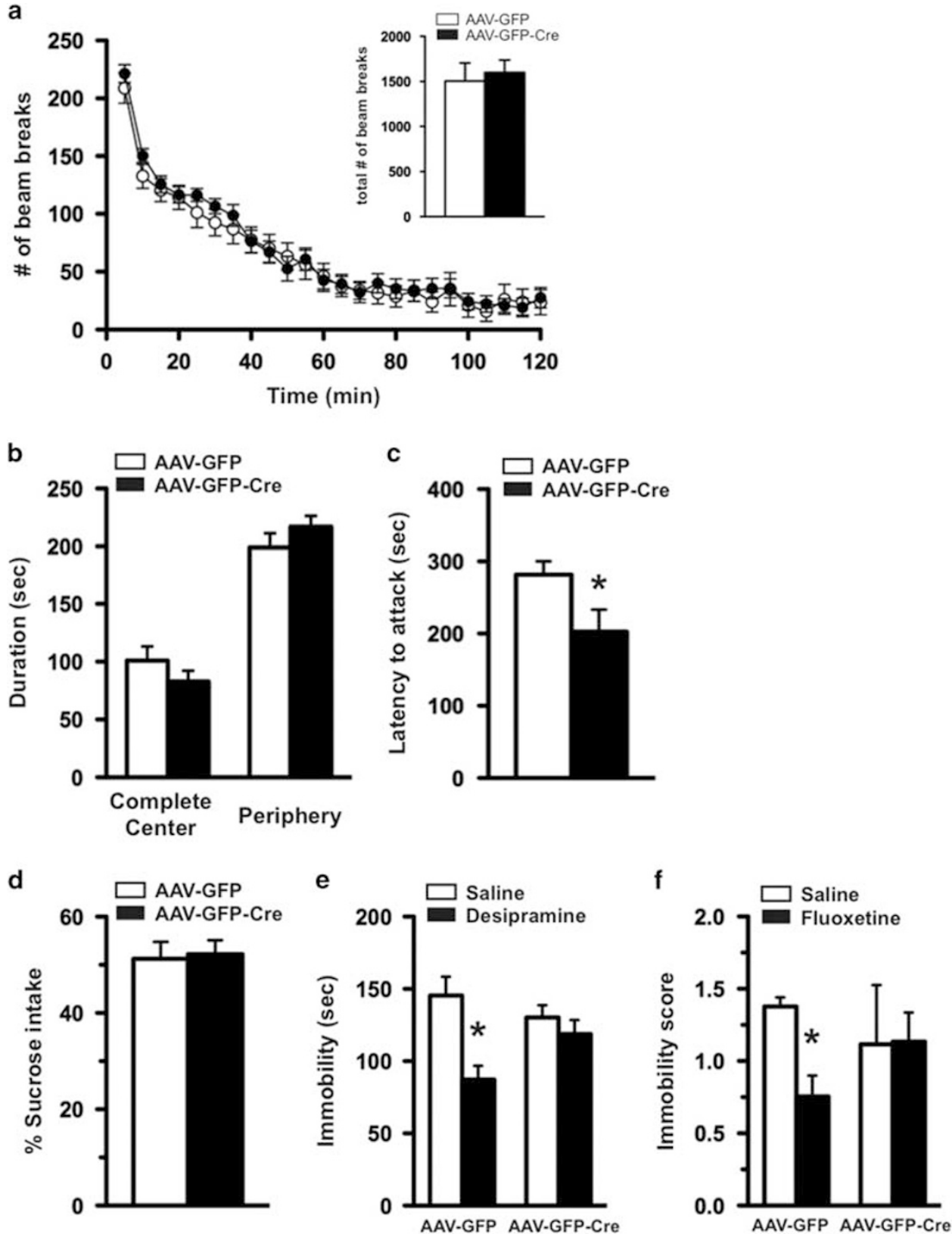

Figure 4 Mice with localized knockdown of tropomyosin receptor kinase B (TrkB) in dorsal raphe nucleus (DRN) display normal locomotor, anxiety-like, and depression-like behavior, but have an attenuated response to antidepressants. (a) Locomotor activity as assessed by the number of horizontal beam breaks over a $2 \mathrm{~h}$ testing period in $5 \mathrm{~min}$ increments. Total beam breaks during the test period were indistinguishable between adeno-associated virus expressing GFP (AAV-GFP) and AAV-GFP tagged to Cre recombinase (AAV-GFP-Cre)-injected fiTrkB mice (see the inset). (b) The animals were assessed for anxiety-like behavior using the open-field test. (c) Aggression-like behavior of flTrkB mice injected with either AAV-GFP or AAV-GFP-Cre was examined in the residentintruder test. Latency to attack the intruder was scored. (d) Depression-like behavior was measured by the sucrose consumption test (AAV-GFP, N= I5; AAV-GFP-Cre, $N=16$ for a, b, c, and d). (e) Antidepressant responses to desipramine were examined by forced swim test (FST). Floxed TrkB mice injected with AAV-GFP, but not AAV-GFP-Cre, showed less immobility in response to sub-chronic treatment with desipramine, in comparison with saline-treated animals (AAV-GFP/saline, $N=8$; AAV-GFP/desipramine, $N=7$; AAV-GFP-Cre/saline, $N=8$; AAV-GFP-Cre/desipramine, $N=8 ;$ two-way ANOVA $\left(F_{1}, 27\right)=\mid 1.24, p=0.0024$ for treatment followed by multiple comparison with $t$-test, $\left.* p<0.05\right)$. ( $f$ ) Chronic treatment with fluoxetine resulted in less immobility in flTrkB mice with AAV-GFP, but not in AAV-GFP-Cre. (AAV-GFP/saline, N=7; AAV-GFP/desipramine, N=7; AAV-GFP-Cre/saline, N=6; AAVGFP-Cre/desipramine, $N=6$; a multiple $t$-test, followed by Holm-Sidak post-hoc analysis to correct for multiple comparisons, revealed that for GFP-SA vs GFPFlX, $p=0.03$, with all other group comparisons are nonsignificant.)

We next examined whether the loss of TrkB in the DRN has an impact on antidepressant responses in the FST. The baseline immobility was similar between saline-treated mice receiving AAV-GFP or AAV-GFP-Cre, suggesting that the loss of TrkB in the DRN did not influence depression-like behavior per se (Figure 4e). In contrast, sub-chronic desipramine treatment resulted in a significant decrease in immobility only in the AAV-GFP-injected mice, whereas AAV-GFP-Cre-injected mice did not respond to the antidepressant effects of desipramine (Figure 4e), suggesting that 
$\operatorname{TrkB}$ in the DRN is required for the antidepressant effects. As desipramine is a tricyclic antidepressant that has effects on both norepinephrine and serotonin, we also examined whether these mice would respond to fluoxetine, a SSRI. Subchronic treatment in which fluoxetine was given 24,4 , and $1 \mathrm{~h}$ before testing did not produce antidepressant responses in either the AAV-GFP- or the AAV-GFP-Cre-injected mice (data not shown). Therefore, we chronically administered fluoxetine for 8 weeks and then tested mice in the FST. Chronic fluoxetine produced a significant decrease in immobility in flTrkB mice injected with AAV-GFP, suggestive of an antidepressant response (Figure 4f). In contrast, mice with the localized deletion of TrkB in the DRN had an attenuated response to fluoxetine.

\section{DISCUSSION}

Here we show that TrkB signaling in the DRN is necessary for antidepressant efficacy. In contrast, knockdown of $B d n f$ selectively in the DRN does not have an impact on antidepressant responses. We also find that $\operatorname{TrkB}$, but not $\mathrm{BDNF}$, in the DRN is involved in aggression behavior. Selective loss of $B d n f$ or TrkB in the DRN did not have an impact on body weight, locomotor activity, anxiety-related behavior, or depression-related behavior, suggesting specificity to the behavioral phenotypes. Collectively, these data reveal an unexpected uncoupling between BDNF-TrkB signaling in the DRN in antidepressant responses and aggression behavior, and strongly suggests that a brain region other than $\mathrm{DRN}$ provides $\mathrm{BDNF}$ for $\operatorname{TrkB}$ function during these behaviors.

In this study, we selectively deleted either $B d n f$ or $\operatorname{Trk} B$, bilaterally in the DRN of adult male mice. We used male mice as loss of $B d n f$ in the brain has been shown to exert differences in behavior between males and females (Autry et $a l, 2009)$. We found that loss of TrkB, but not Bdnf, in the DRN resulted in increased aggressive behavior. These data are unexpected as BDNF heterozygous knockouts, as well as forebrain-specific BDNF knockouts, display increased aggressive behavior (Lyons et al, 1999; Rios et al, 2001). Previous work has shown that enhanced aggression observed in BDNF heterozygous knockout mice is rescued by overexpression of the truncated TrkB receptor, whose physiological role is believed to negatively regulate BDNF (Saarelainen et al, 2003). Serotonin has been shown to be a key neurotransmitter in aggressive behavior with serotonergic neurons in the DRN as a key driver of these behaviors (Takahashi et al, 2015; Takahashi and Miczek, 2014). Taken together, the previously reported increased aggression of the $B d n f$ heterozygous mice, combined with the present data showing $\operatorname{TrkB}$ in the $\mathrm{DRN}$ is required for aggressive behavior, suggests that BDNF is released from a source extrinsic to DRN to activate TrkB receptors and mediate effects on aggression.

We explored whether the selective loss of $B d n f$ or $\operatorname{Trk} B$ in the DRN would have an impact on depression-related behavior or antidepressant responses. BDNF can exert differing effects on depression-related behavior depending on the brain region, such as the nucleus accumbens (Eisch et al, 2003), ventral tegmental area (Berton et al, 2006), or hippocampus, highlighting the importance of examining the impact of BDNF in specific brain regions. Previous work has shown that BDNF in forebrain regions (Monteggia et al, 2004), specifically the dentate gyrus region of the hippocampus (Adachi et al, 2008), is required for antidepressant responses. The viral-mediated approach in the current study resulted in a $\sim 70 \%$ deletion of $B d n f$ mRNA in the DRN. However, the regional-specific knockdown of $B d n f$ in the DRN did not alter 'depression-related' behavior as assessed in the sucrose consumption test and the FST. These paradigms are widely used to examine depression-like behavior and antidepressant responses in a variety of genetic models (Cryan et al, 2002), although may be more reliable as a screen for antidepressant responses (Petit-Demouliere et al, 2005). Nevertheless, the loss of BDNF in the DRN did not produce behavioral changes in sucrose consumption or FST behavior. The robust deletion of $B d n f$ also did not have an impact on antidepressant efficacy, suggesting that BDNF originating from the DRN is not involved in antidepressant responses.

In contrast to the BDNF findings, the current study reveals a critical cell autonomous role for $\operatorname{TrkB}$ in the DRN in antidepressant responses. $\operatorname{TrkB}$ is the high-affinity receptor for BDNF (Soppet et al, 1991), suggesting that BDNF acting on $\operatorname{TrkB}$ receptors in the DRN mediate antidepressant responses. However, neurtrophin-3 (NT-3) and NT-4 are also ligands of TrkB. Haenisch et al (2009) reported that hippocampal expression of NT-3 is attenuated by chronic stress, which is reversed by chronic antidepressant treatments using a norepinephrine transporter inhibitor. However, there is currently no direct data demonstrating a necessity of NT-3 in the brain to mediate antidepressant efficacy. Similarly, the roles of NT-4 in depression-like behavior as well as antidepressant responses are largely unknown; yet, it is unlikely that NT-4 can replace BDNF functions given the distinct neuronal impairments found in $B d n f$ and NT-4-null mice (Conover et al, 1995; Liu et al, 1995). Moreover, previous work has shown that NT-4 and BDNF possess unique downstream signaling pathways on TrkB activation, suggesting these growth factors are not functionally redundant (Fan et al, 2000). Taken together, these data suggest that secreted BDNF is the ligand acting on TrkB receptors in the DRN to mediate antidepressant efficacy.

In prior work from our laboratory, we demonstrated that BDNF in the dentate gyrus, but not the CA1 region of the hippocampus, was required for antidepressant efficacy (Adachi et al, 2008). It is, however, an open question as to where $\mathrm{BDNF}$ is released to activate $\operatorname{TrkB}$ receptor in the DRN to convey antidepressant responses. The dentate gyrus is innervated by serotonergic neurons projecting from the DRN (Adachi et al, 2009; Monteggia et al, 2004), consistent with the idea that BDNF secreted from the dentate gyrus is activating $\mathrm{TrkB}$ receptors on $\mathrm{DRN}$ neurons to mediate antidepressant efficacy. Indeed, several studies have shown interactions between $\mathrm{BDNF}$ and serotonergic neurons originating in the raphe. BDNF can promote sprouting of serotonergic axons (Mamounas et al, 1995) as well as increase the firing rate of serotonergic neurons in the raphe (Celada et al, 1996). Previous work has also shown that TrkB mRNA is expressed in the cell bodies of serotonergic neurons in the raphe (Soppet et al, 1991). Moreover, use of a serotonin-specific neurotoxin to lesion serotonergic cells 
projecting from the raphe results in a decrease in $\operatorname{TrkB}$ expression in these cells without alterations in $\operatorname{TrkB}$ expression in the target region, the hippocampus, demonstrating the presence of $\operatorname{TrkB}$ receptors on serotonergic neurons from the DRN (Madhav et al, 2001) and providing a possible explanation for how BDNF has an impact on serotonergic neurons. Rather intriguingly, there seems to also be a reciprocal interaction between $\mathrm{BDNF}$ and serotonin as antidepressants that increase serotonin increase BDNF expression in forebrain regions, including the hippocampus (Altar et al, 2003; Nibuya et al, 1995). Collectively, these results suggest a model whereby increased serotonergic tone after antidepressant administration may upregulate BDNF release in the dentate gyrus, which in turn activates TrkB receptors in the DRN resulting in a positive feedback loop. Future studies will be necessary to test this hypothesis, as well as determine which intracellular signaling pathway is involved in BDNF-TrkB-mediated antidepressant responses in the DRN.

In summary, our data demonstrates that selective loss of $\operatorname{TrkB}$ in the DRN results in an attenuated response to classical antidepressants and heightened aggression. These findings reveal separate roles for BDNF and TrkB in the DRN as they relate to antidepressant effects and highlight an important distinction when extrapolating BDNF effects to that of TrkB receptors in the CNS. These novel findings further reveal a cell autonomous role for TrkB in the DRN in antidepressant responses and demonstrate that BDNF extrinsic to the DRN is required for antidepressant effects.

\section{FUNDING AND DISCLOSURE}

AEA, MM, and KS report no financial or potential conflicts of interest.

\section{ACKNOWLEDGMENTS}

We thank Dr Luis Parada for providing flTrkB mice and Dr Rudolf Jaenisch for providing flBdnf mice. We also thank Dr Ege Kavalali and members of the Monteggia lab for helpful comments and suggestions. This work was supported by National Institute of Health grant MH070727 (LMM), by a Brain and Behavior Foundation Award (LMM) and the International Mental Health Research Organization (LMM). MA is currently employed with Astellas Research Institute of America. LMM reports having received lecture fees from Shire Pharmaceuticals.

\section{REFERENCES}

Adachi M, Autry AE, Covington HE 3rd, Monteggia LM (2009). MeCP2-mediated transcription repression in the basolateral amygdala may underlie heightened anxiety in a mouse model of Rett syndrome. J Neurosci 29: 4218-4227.

Adachi M, Barrot M, Autry AE, Theobald D, Monteggia LM (2008). Selective loss of brain-derived neurotrophic factor in the dentate gyrus attenuates antidepressant efficacy. Biol Psychiatry 63: 642-649.

Altar CA, Whitehead RE, Chen R, Wortwein G, Madsen TM (2003). Effects of electroconvulsive seizures and antidepressant drugs on brain-derived neurotrophic factor protein in rat brain. Biol Psychiatry 54: 703-709.
Autry AE, Adachi M, Cheng P, Monteggia LM (2009). Genderspecific impact of brain-derived neurotrophic factor signaling on stress-induced depression-like behavior. Biol Psychiatry 66: 84-90.

Berton O, McClung CA, Dileone RJ, Krishnan V, Renthal W, Russo SJ et al (2006). Essential role of BDNF in the mesolimbic dopamine pathway in social defeat stress. Science 311: 864-868.

Bjorkholm C, Monteggia LM (2015). BDNF - a key transducer of antidepressant effects. Neuropharmacology 102: 72-79.

Burke TF, Advani T, Adachi M, Monteggia LM, Hensler JG (2013). Sensitivity of hippocampal 5-HT1A receptors to mild stress in BDNF-deficient mice. Int J Neuropsychopharmacol 16: 631-645.

Castren E, Rantamaki T (2010). The role of BDNF and its receptors in depression and antidepressant drug action: Reactivation of developmental plasticity. Dev Neurobiol 70: 289-297.

Celada P, Siuciak JA, Tran TM, Altar CA, Tepper JM (1996). Local infusion of brain-derived neurotrophic factor modifies the firing pattern of dorsal raphe serotonergic neurons. Brain Res 712: 293-298.

Conover JC, Erickson JT, Katz DM, Bianchi LM, Poueymirou WT, McClain J et al (1995). Neuronal deficits, not involving motor neurons, in mice lacking BDNF and/or NT4. Nature 375: 235-238.

Cryan JF, Markou A, Lucki I (2002). Assessing antidepressant activity in rodents: recent developments and future needs. Trends Pharmacol Sci 23: 238-245.

Duman RS, Monteggia LM (2006). A neurotrophic model for stressrelated mood disorders. Biol Psychiatry 59: 1116-1127.

Eisch AJ, Bolanos CA, de Wit J, Simonak RD, Pudiak CM, Barrot M et al (2003). Brain-derived neurotrophic factor in the ventral midbrain-nucleus accumbens pathway: a role in depression. Biol Psychiatry 54: 994-1005.

Fan G, Egles C, Sun Y, Minichiello L, Renger JJ, Klein R et al (2000). Knocking the NT4 gene into the BDNF locus rescues BDNF deficient mice and reveals distinct NT4 and BDNF activities. Nat Neurosci 3: 350-357.

Gourley SL, Wu FJ, Taylor JR (2008). Corticosterone regulates pERK1/2 map kinase in a chronic depression model. Ann NY Acad Sci 1148: 509-514.

Griebel G, Belzung C, Perrault G, Sanger DJ (2000). Differences in anxiety-related behaviours and in sensitivity to diazepam in inbred and outbred strains of mice. Psychopharmacology (Berl) 148: $164-170$

Haenisch B, Bilkei-Gorzo A, Caron MG, Bonisch H (2009). Knockout of the norepinephrine transporter and pharmacologically diverse antidepressants prevent behavioral and brain neurotrophin alterations in two chronic stress models of depression. J Neurochem 111: 403-416.

Hensler JG, Advani T, Monteggia LM (2007). Regulation of serotonin-1A receptor function in inducible brain-derived neurotrophic factor knockout mice after administration of corticosterone. Biol Psychiatry 62: 521-529.

Hoshaw BA, Malberg JE, Lucki I (2005). Central administration of IGF-I and BDNF leads to long-lasting antidepressant-like effects. Brain Res 1037: 204-208.

Kessler RC, Berglund P, Demler O, Jin R, Koretz D, Merikangas KR et al (2003). The epidemiology of major depressive disorder: results from the National Comorbidity Survey Replication (NCSR). JAMA 289: 3095-3105.

Lesch KP (2004). Gene-environment interaction and the genetics of depression. J Psychiatry Neurosci 29: 174-184.

Lindholm JS, Castren E (2014). Mice with altered BDNF signaling as models for mood disorders and antidepressant effects. Front Behav Neurosci 8: 143. 
Liu X, Ernfors P, Wu H, Jaenisch R (1995). Sensory but not motor neuron deficits in mice lacking NT4 and BDNF. Nature 375: 238-241.

Luikart BW, Nef S, Virmani T, Lush ME, Liu Y, Kavalali ET et al (2005). TrkB has a cell-autonomous role in the establishment of hippocampal Schaffer collateral synapses. J Neurosci 25: 3774-3786.

Lunden JW, Kirby LG (2013). Opiate exposure and withdrawal dynamically regulate mRNA expression in the serotonergic dorsal raphe nucleus. Neuroscience 254: 160-172.

Lyons WE, Mamounas LA, Ricaurte GA, Coppola V, Reid SW, Bora SH et al (1999). Brain-derived neurotrophic factor-deficient mice develop aggressiveness and hyperphagia in conjunction with brain serotonergic abnormalities. Proc Natl Acad Sci USA 96: 15239-15244.

Madhav TR, Pei Q, Zetterstrom TS (2001). Serotonergic cells of the rat raphe nuclei express mRNA of tyrosine kinase B (trkB), the high-affinity receptor for brain derived neurotrophic factor (BDNF). Brain Res Mol Brain Res 93: 56-63.

Mamounas LA, Blue ME, Siuciak JA, Altar CA (1995). Brain-derived neurotrophic factor promotes the survival and sprouting of serotonergic axons in rat brain. J Neurosci 15: 7929-7939.

McDevitt RA, Neumaier JF (2011). Regulation of dorsal raphe nucleus function by serotonin autoreceptors: a behavioral perspective. J Chem Neuroanat 41: 234-246.

Monteggia LM, Barrot M, Powell CM, Berton O, Galanis V, Gemelli $\mathrm{T}$ et al (2004). Essential role of brain-derived neurotrophic factor in adult hippocampal function. Proc Natl Acad Sci USA 101: 10827-10832.

Nibuya M, Morinobu S, Duman RS (1995). Regulation of BDNF and trkB mRNA in rat brain by chronic electroconvulsive seizure and antidepressant drug treatments. J Neurosci 15: 7539-7547.

Petit-Demouliere B, Chenu F, Bourin M (2005). Forced swimming test in mice: a review of antidepressant activity. Psychopharmacology (Berl) 177: 245-255.
Porsolt RD, Le Pichon M, Jalfre M (1977). Depression: a new animal model sensitive to antidepressant treatments. Nature 266: 730-732.

Rios M, Fan G, Fekete C, Kelly J, Bates B, Kuehn R et al (2001). Conditional deletion of brain-derived neurotrophic factor in the postnatal brain leads to obesity and hyperactivity. Mol Endocrinol 15: 1748-1757.

Saarelainen T, Hendolin P, Lucas G, Koponen E, Sairanen M, MacDonald E et al (2003). Activation of the TrkB neurotrophin receptor is induced by antidepressant drugs and is required for antidepressant-induced behavioral effects. J Neurosci 23: 349-357.

Shirayama Y, Chen AC, Nakagawa S, Russell DS, Duman RS (2002). Brain-derived neurotrophic factor produces antidepressant effects in behavioral models of depression. J Neurosci 22: 3251-3261.

Siuciak JA, Boylan C, Fritsche M, Altar CA, Lindsay RM (1996). $\mathrm{BDNF}$ increases monoaminergic activity in rat brain following intracerebroventricular or intraparenchymal administration. Brain Res 710: 11-20.

Siuciak JA, Clark MS, Rind HB, Whittemore SR, Russo AF (1998). BDNF induction of tryptophan hydroxylase mRNA levels in the rat brain. J Neurosci Res 52: 149-158.

Soppet D, Escandon E, Maragos J, Middlemas DS, Reid SW, Blair J et al (1991). The neurotrophic factors brain-derived neurotrophic factor and neurotrophin-3 are ligands for the trkB tyrosine kinase receptor. Cell 65: 895-903.

Takahashi A, Lee RX, Iwasato T, Itohara S, Arima H, Bettler B et al (2015). Glutamate input in the dorsal raphe nucleus as a determinant of escalated aggression in male mice. J Neurosci 35: 6452-6463.

Takahashi A, Miczek KA (2014). Neurogenetics of aggressive behavior: studies in rodents. Curr Top Behav Neurosci 17: 3-44.

Tork I (1990). Anatomy of the serotonergic system. Ann NY Acad Sci 600: 9-34; discussion 34-35.

Waterhouse EG, Xu B (2009). New insights into the role of brain-derived neurotrophic factor in synaptic plasticity. Mol Cell Neurosci 42: 81-89. 